\title{
Intracorporeal gastrojejunostomy in Roux-en-Y reconstruction with its efferent loop located on the patient's left side following totally laparoscopic distal gastrectomy: A case report
}

\author{
TAKAYA TOKUHARA ${ }^{1,2}$, EIJI NAKATA ${ }^{2}$, AKIHITO OGATA ${ }^{1}$, TOSHIYUKI TENJO ${ }^{2}$, \\ ISAO KAWAI $^{2}$, KEISAKU KONDO ${ }^{2}$, SHIGERU HATABE ${ }^{2}$ and KAZUTAKE YOKOYAMA $^{1}$ \\ ${ }^{1}$ Department of Surgery, Tanigawa Memorial Hospital, Osaka 567-0031; \\ ${ }^{2}$ Department of Surgery, Otori Stomach and Intestines Hospital, Osaka 593-8311, Japan
}

Received October 26, 2021; Accepted December 8, 2021

DOI: $10.3892 / \mathrm{mco} .2021 .2480$

\begin{abstract}
The drawback of intracorporeal gastrojejunostomy using only endoscopic linear staplers in antecolic Roux-en-Y (R-Y) reconstruction with its efferent loop located on the patient's left side following totally laparoscopic distal gastrectomy (TLDG) is the occurrence of anastomotic failure, even though this reconstruction system is assumed to prevent intraoperative and postoperative twisting of the gastrojejunostomy and lifted jejunum. This case report presents two patients with gastric cancer who underwent intracorporeal gastrojejunostomy consisting of linear stapling and hand suturing in antecolic R-Y reconstruction with its efferent loop located on the patient's left side following TLDG to prevent anastomotic failure of the gastrojejunostomy. After the sacrificed jejunum was created, linear stapling of the greater curvature of the remnant stomach and the lifted jejunum without dividing the jejunum was performed. After removing the sacrificed jejunum and creating a good view of the posterior side of the stapler entry hole, the stapler entry hole was closed from the posterior side to the anterior side, using a single-layer full-thickness and serosubmucosal hand suturing technique with knotted sutures and a knotless barbed suture. No anastomotic failure of the gastrojejunostomy occurred in either patient. Intracorporeal gastrojejunostomy consisting of linear stapling and hand suturing could be an option for
\end{abstract}

Correspondence to: Dr Takaya Tokuhara, Department of Surgery, Tanigawa Memorial Hospital, 1-16-59 Kasuga, Ibaraki, Osaka 567-0031, Japan

E-mail: takaya.tokuhara@ompu.ac.jp

Abbreviations: B-II, Billroth-II; R-Y, Roux-en-Y; TLDG, totally laparoscopic distal gastrectomy

Key words: totally laparoscopic distal gastrectomy, Roux-en-Y reconstruction, intracorporeal gastrojejunostomy, anastomotic failure gastrojejunostomy in antecolic R-Y reconstruction with its efferent loop located on the patient's left side following TLDG because it can aid in the prevention of anastomotic failure.

\section{Introduction}

Over the past 20 years, totally laparoscopic distal gastrectomy (TLDG), in which all procedures including lymph node dissection, resection of the stomach and duodenum, and anastomosis are performed intracorporeally, has been developed due to improvements in the instruments and techniques. Roux-en-Y (R-Y) reconstruction following TLDG is performed when the remnant stomach is small, the length of the duodenal bulb is short, or gastroesophageal reflux due to hiatal hernia is anticipated (1-8). Several authors reported the performance of antecolic R-Y reconstruction with its efferent loop located on the patient's left side following TLDG combined with the gastrojejunostomy, in which a functional end-to-end anastomosis is created using endoscopic linear staplers $(1,4,5)$. In this gastrojejunostomy technique, first, without dividing the lifted jejunum, stapling of the greater curvature of the remnant stomach and the lifted jejunum without creating a gap using one linear stapler is performed so that the distal side of the lifted jejunum is located on the patient's left side. Thereafter, closure of the stapler entry hole and division of the lifted jejunum are carried out simultaneously by a second stapling. However, one drawback of this gastrojejunostomy technique is the occurrence of anastomotic failure at the closing staple line due to slippage of the alimentary tract during the second stapling (4), even though antecolic R-Y reconstruction with its efferent loop located on the patient's left side following TLDG is assumed to be useful thanks to the prevention of intraoperative and postoperative twisting of the gastrojejunostomy and lifted jejunum (9). Here, we describe our initial experience in two patients with a novel gastrojejunostomy technique consisting of linear stapling and hand suturing in antecolic R-Y reconstruction with its efferent loop located on the patient's left side following TLDG to prevent anastomotic failure of the gastrojejunostomy. 


\section{Patients and methods}

Patients and ethical approval. Two patients with gastric cancer underwent intracorporeal gastrojejunostomy consisting of linear stapling and hand suturing in antecolic R-Y reconstruction with its efferent loop located on the patient's left side following TLDG. Written informed consent was obtained from each patient, both of whom agreed to undergo TLDG. Case 1 (73-year-old female) was operated on at Otori Stomach and Intestines Hospital (Sakai-City, Osaka, Japan) in August 2019, and Case 2 (64-year-old male) was operated on at Tanigawa Memorial Hospital (Ibaraki-City, Osaka, Japan) in March 2021. This procedure was approved by the Institutional Review Board of the Otori Stomach and Intestines Hospital (approval no. 19000010) and Tanigawa Memorial Hospital (approval no. 21000001).

Procedure. The patients were placed in the modified lithotomy position. The operator stood between the patient's legs, with the first assistant manipulating a laparoscope at the patient's left side and the second assistant at the right side (Fig. 1). After five ports were placed in the upper abdomen including the umbilicus, a Nathanson's retractor was inserted from just below the xiphoid process to elevate the round ligament and the lateral segment of the liver (Fig. 1). Because the endoscopic linear stapler was inserted through the left lower port, all staplings were performed by the right hand of the operator positioned between the patient's legs. LDG with D1+ lymphadenectomy based on the Japanese treatment guidelines was performed under a pneumoperitoneum (10). The duodenal bulb was transected using one endoscopic linear stapler $(60 \mathrm{~mm}$ Gold cartridge, Powered ECHELON FLEX ${ }^{\circledR}$ GST SYSTEM; Ethicon, Cincinnati, OH, USA) during lymphadenectomy. The proximal stomach was transected using two linear staplers (60 mm Gold cartridge, Powered ECHELON FLEX ${ }^{\circledR}$ GST SYSTEM; Ethicon) after lymphadenectomy, referring to preoperative endoscopic markings (11). The specimen was retrieved through the extended umbilical wound using a large plastic bag, after which pneumoperitoneum was re-established.

Antecolic R-Y reconstruction with its efferent loop located on the patient's left side was then performed in the two patients. This technique consisted of intracorporeal gastrojejunostomy and extracorporeal jejunojejunostomy with the use of linear stapling and hand suturing. The jejunum was grasped intracorporeally so that the distal side was located on the patient's left side, and the mesentery was dissected along the lifted jejunum from a portion $20 \mathrm{~cm}$ distal to the ligament of Treitz to a portion $30 \mathrm{~cm}$ distal. Small incisions were made on the antimesenteric side of the lifted jejunum $33 \mathrm{~cm}$ distal to the ligament of Treitz and the greater curvature side of the remnant stomach. The operator inserted one linear stapler $(60 \mathrm{~mm}$ Gold cartridge, Powered ECHELON FLEX ${ }^{\circledR}$ GST SYSTEM, Ethicon) into the jejunum toward the distal side. Then, the operator retracted the jejunum antecolically and inserted the stapler into the remnant stomach (Fig. 2). Linear stapling of the greater curvature of the remnant stomach and the antimesenteric surface of the lifted jejunum was performed without creating a gap (Fig. 2). The operator transected the lifted jejunum at the portions $20 \mathrm{~cm}$ and $30 \mathrm{~cm}$ distal to the ligament of Treitz using one linear stapler $\left(60 \mathrm{~mm}\right.$, White cartridge, Powered ECHELON FLEX ${ }^{\circledast}$

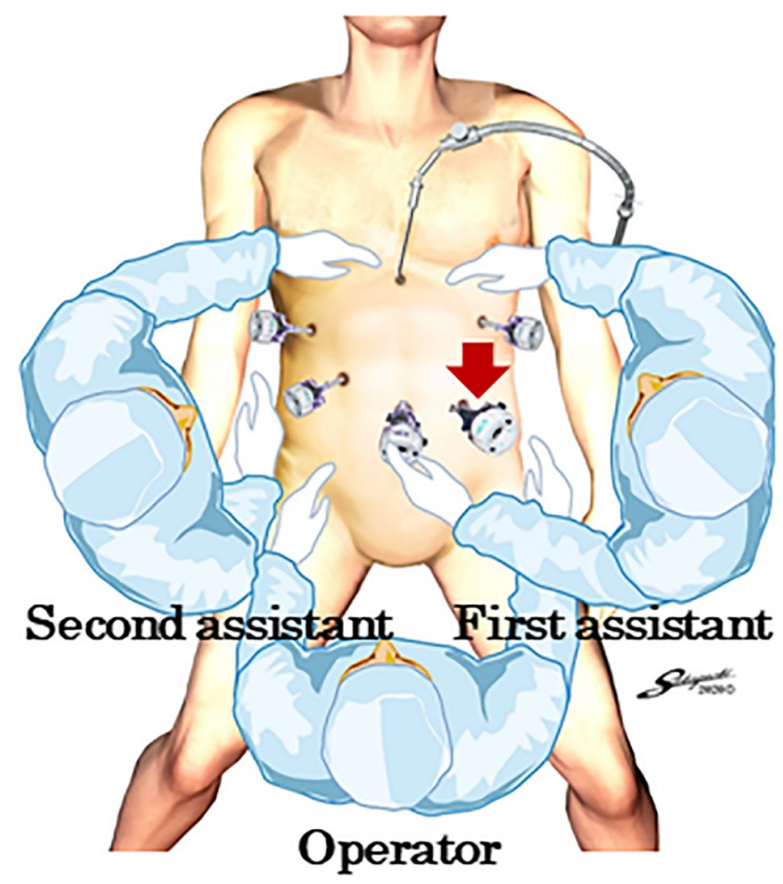

Figure 1. Port placements and positions of the operator and assistants. The operator inserted the stapler through the left lower port (red arrow), and all staplings were performed by the right hand of the operator.

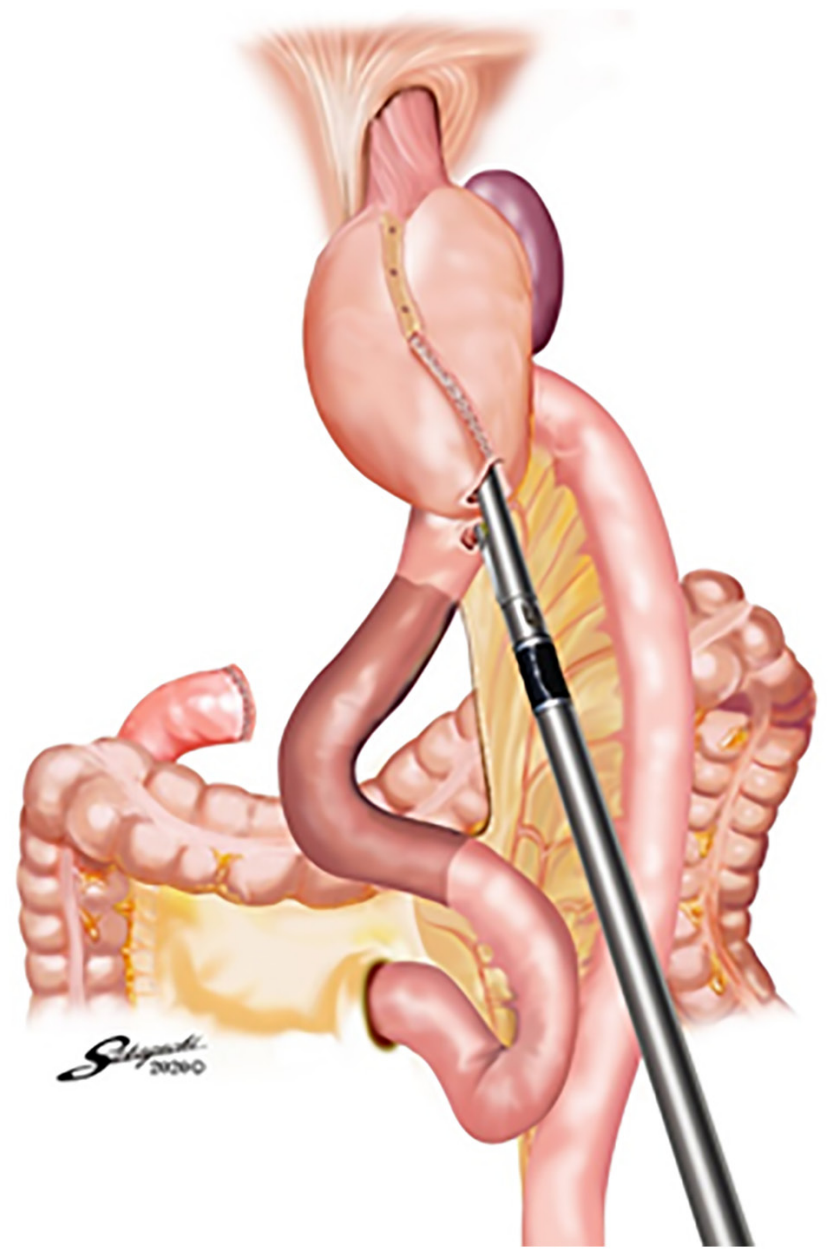

Figure 2. Stapling of the remnant stomach and lifted jejunum. Linear stapling of the greater curvature of the remnant stomach and the antimesenteric surface of the lifted jejunum without creating a gap was performed. 

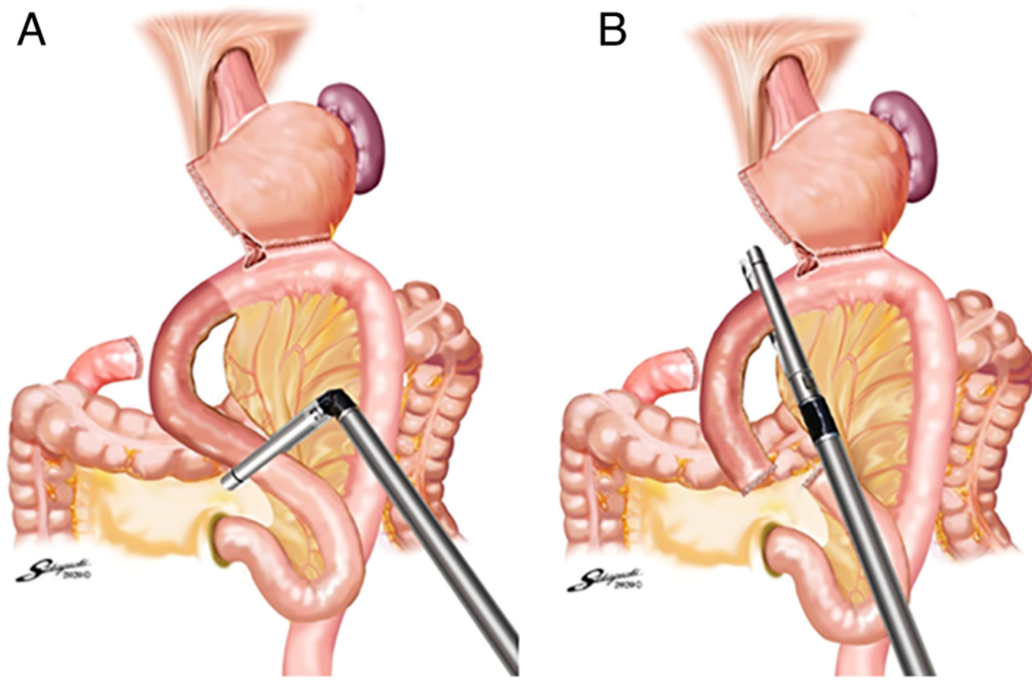

Figure 3. Transection of the lifted jejunum. (A) The lifted jejunum was transected at a portion $20 \mathrm{~cm}$ distal to the ligament of Treitz using one linear stapler. (B) The lifted jejunum was then transected at a portion $30 \mathrm{~cm}$ distal to the ligament of Treitz using another linear stapler.

A

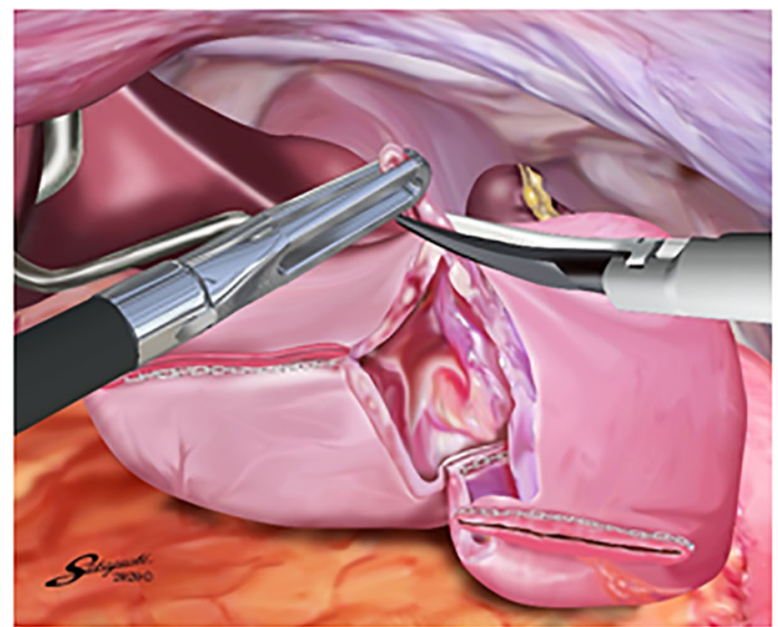

$\mathrm{B}$

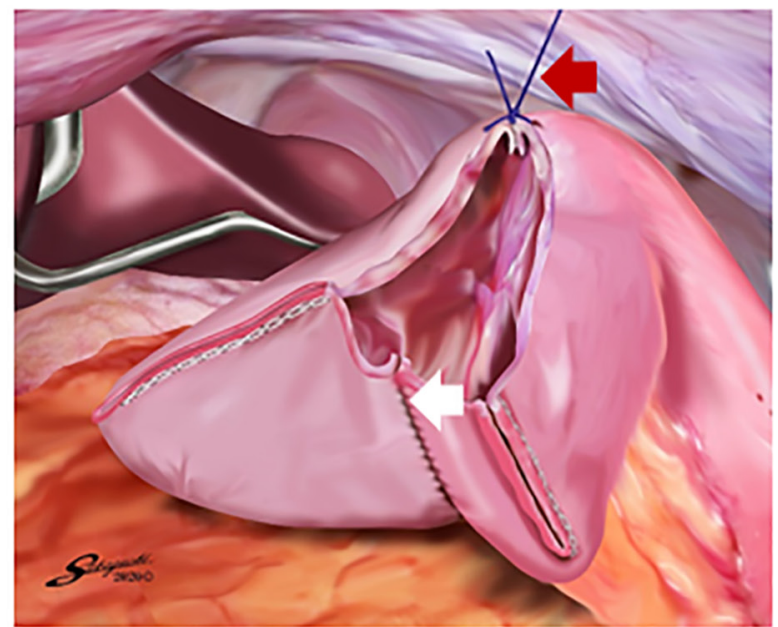

Figure 4. Creation of a good view of the posterior side of the stapler entry hole. (A) The edge of the anterior side of the stapler entry hole was removed. (B) To create a good view of the posterior side (white arrow), the full-thickness knotted suture of 3-0 Prolene at the anterior curvature side (red arrow) was retracted toward the ventral side.

GST SYSTEM, Ethicon) at each location (Fig. 3A and B), and the sacrificed jejunum was removed in a cut finger of a surgical glove.

The operator closed the stapler entry hole with the right hand using a single-layer full-thickness, serosubmucosal suturing technique with knotted sutures and a knotless barbed suture after confirming that there was no bleeding in the intraluminal staple line. After removing the edge of the anterior side of the stapler entry hole, a full-thickness suture of 3-0 Prolene (Ethicon) using the extracorporeal slip knot technique (Roeder's knot) was placed on the anterior side of the stapler entry hole (Fig. 4A). The second assistant retracted this knotted suture toward the ventral side to create a good view of the posterior side of the stapler entry hole (Fig. 4B). Single-layer full-thickness and serosubmucosal continuous suturing with a 15-cm-long absorbable barbed suture (3-0 V-Loc 180; VLOCL0604; taper point, 1/2 circle/26 mm; Covidien) was performed from the posterior side to the anterior side. The first full-thickness stitch was placed on the posterior side, and the needle was passed through the loop (Fig. 5A). The second stitch was made between the serosubmucosal layers of the lifted jejunum and remnant stomach (Fig. 5B and C). This single-layer serosubmucosal continuous suturing with a 15-cm-long absorbable barbed suture (3-0 V-Loc 180) was performed until the last stitch was made (Fig. 5D). The last full-thickness stitch was placed on the anterior side so that the suture crossed over the knotted 3-0 Prolene suture (Fig. 5E). The suture end was cut simply to be as short as possible. Some full-thickness knotted sutures of 3-0 Prolene were added at the site with a broad pitch (Fig. 5F). Thereafter, the gastrojejunostomy in antecolic R-Y reconstruction with its efferent loop located on the patient's left side was completed (Fig. 6A-C).

After a wound retractor (Alexis Wound Retractor S, Applied Medical) was attached to the extended umbilical wound, a side-to-side jejunojejunostomy with the use of linear stapling and hand suturing was performed extracorporeally 
A

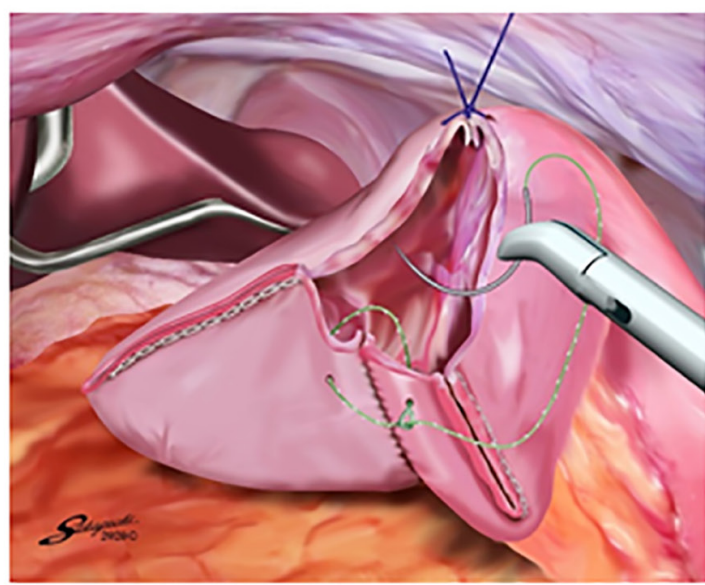

C

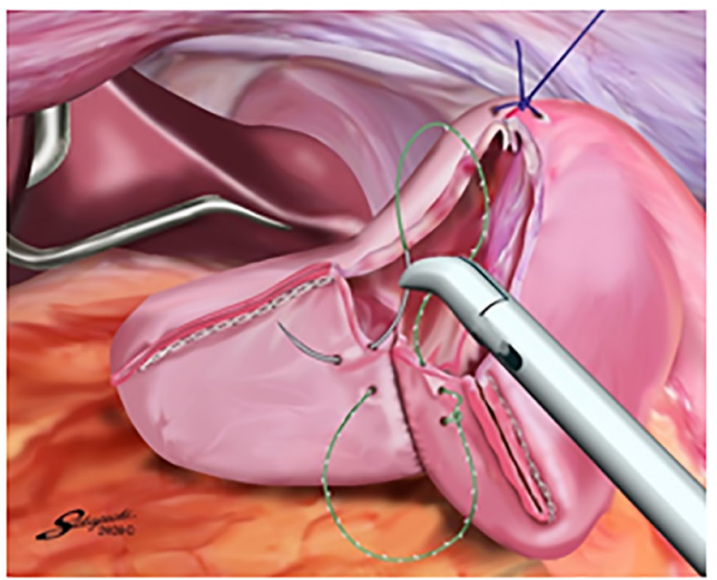

E

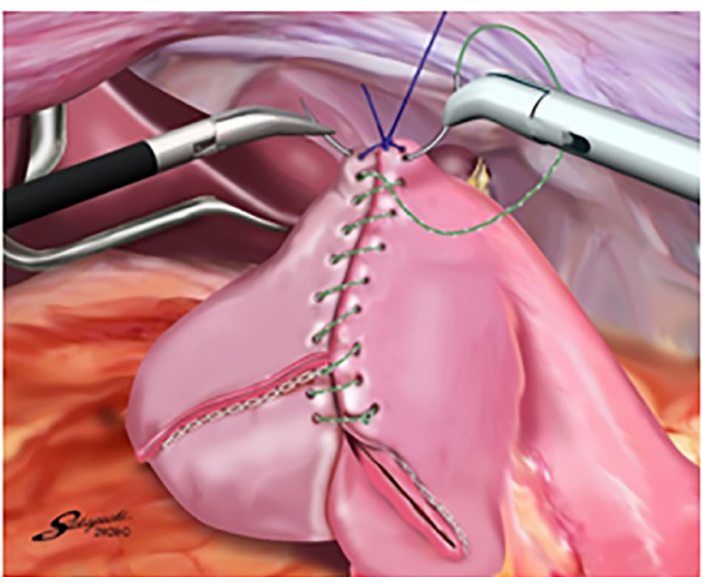

$\mathrm{B}$

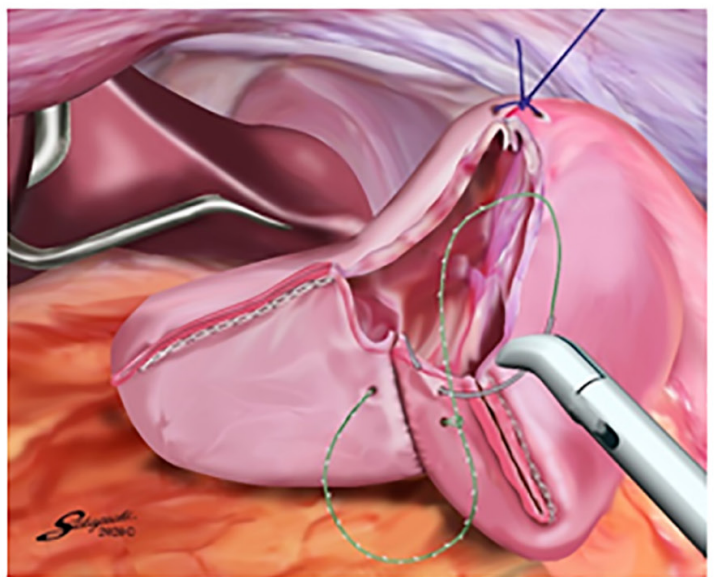

D

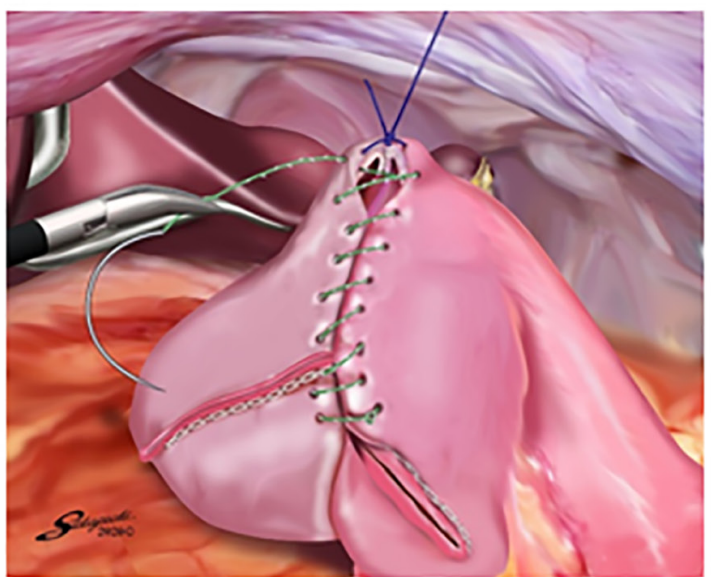

$\mathrm{F}$

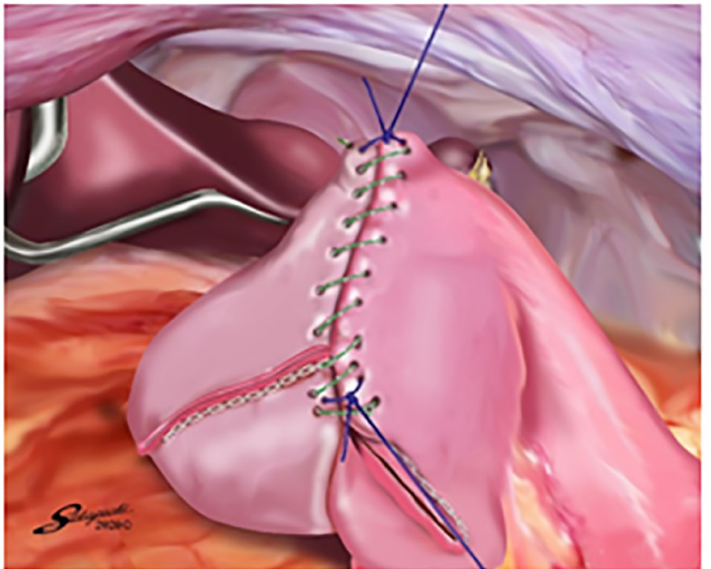

Figure 5. Single-layer, full-thickness and serosubmucosal hand suturing technique of the stapler entry hole. (A) The first full-thickness stitch with a 15-cm long 3-0 V-Loc 180 was placed on the posterior side, and the needle was passed through the loop. (B) The second stitch with the 15 -cm long 3-0 V-Loc 180 was made on the serosubmucosal layer of the lifted jejunum. (C) The second stitch with the 15-cm long 3-0 V-Loc 180 was made on the serosubmucosal layer of the remnant stomach. (D) Single-layer serosubmucosal continuous suturing of the stapler entry hole with the 15-cm long 3-0 V-Loc 180 was performed until the last stitch was made. (E) The last full-thickness stitch with the 15-cm long 3-0 V-Loc 180 was placed on the anterior side so that the suture crossed over the knotted 3-0 Prolene suture. (F) Several full-thickness knotted 3-0 Prolene sutures using the extracorporeal slip knot technique (Roeder's knot) were added at the site with a broad pitch.

to create a $30-\mathrm{cm}$ R-Y limb. No stitches needed to be placed in the duodenal stump and the antimesenteric side of the lifted jejunum. Petersen's defect and the jejunojejunal mesenteric defect were closed intracorporeally with two 15-cm-long nonabsorbable barbed sutures (3-0 V-Loc PBT; VLOCN0604; taper point, 1/2 circle/26 mm; Covidien) (12). Finally, the antecolic R-Y reconstruction with its efferent loop located on the patient's left side following TLDG was completed (Fig. 7).

\section{Results}

No twisting of the gastrojejunostomy and lifted jejunum was encountered intraoperatively in either patient (Fig. 7), nor 
A

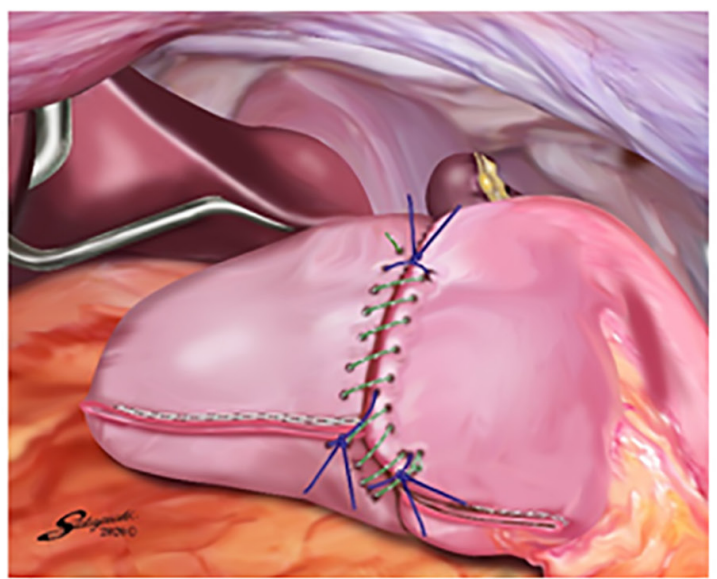

B

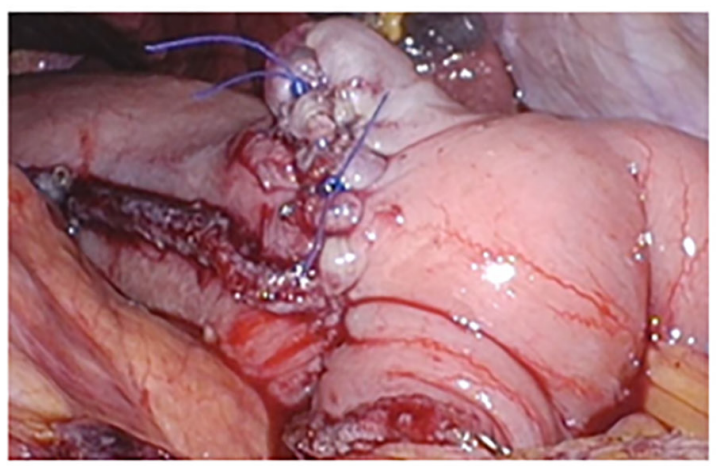

C

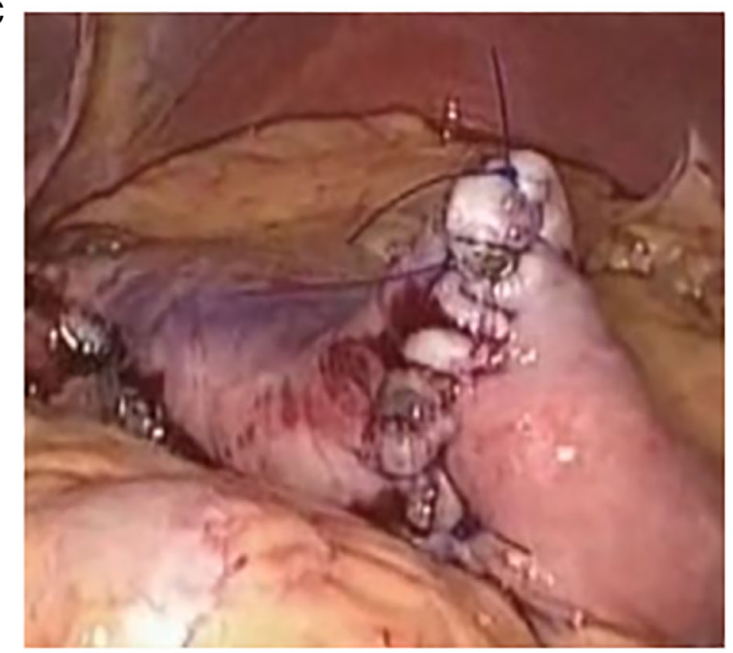

Figure 6. Gastrojejunostomy with linear stapling and hand suturing. (A) Gastrojejunostomy with linear stapling and hand suturing. The gastrojejunostomy with linear stapling and hand suturing in antecolic Roux-en-Y reconstruction with its efferent loop located on the patient's left side was completed in (B) case 1 and (C) case 2.

were there any postoperative complications including anastomotic failure, anastomotic stenosis, stasis, or pancreatic fistula. Upper gastrointestinal fluoroscopic images on postoperative day 10 show no anastomotic failure or stenosis of the gastrojejunostomy in either patient (Fig. 8A and B). The follow-up period was 25.8 months in Case 1 and 7.9 months in Case 2. The efferent loop in both patients was confirmed to be located on the patient's left side by endoscopic examination 3 months after the operation along with confirmation that no twisting of the gastrojejunostomy and lifted jejunum had occurred postoperatively (Fig. 9A and B). Moreover,

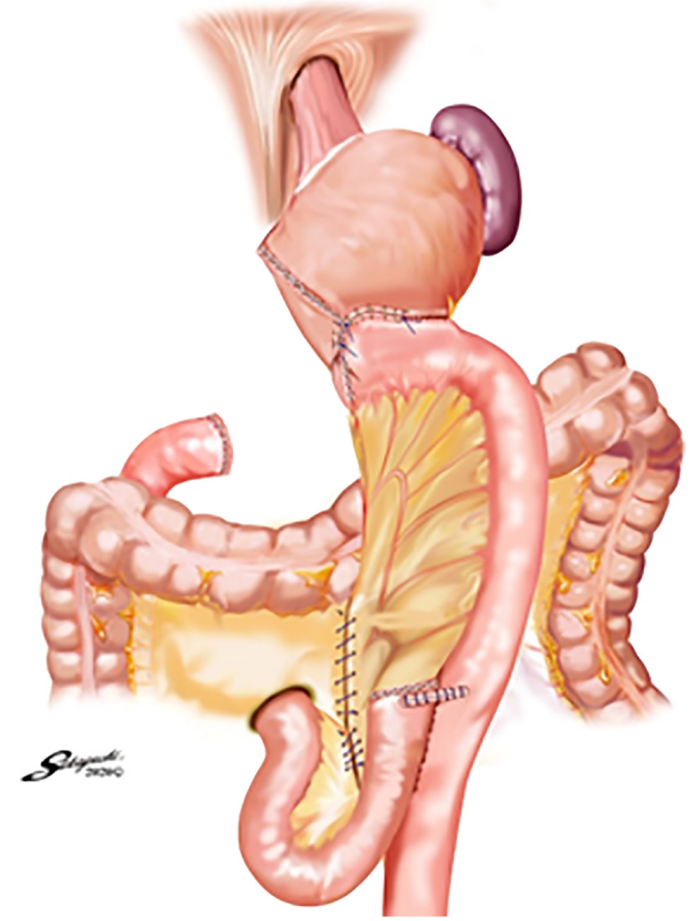

Figure 7. Antecolic Roux-en-Y reconstruction with its efferent loop located on the patient's left side. There was no twisting of the gastrojejunostomy and lifted jejunum intraoperatively.
A

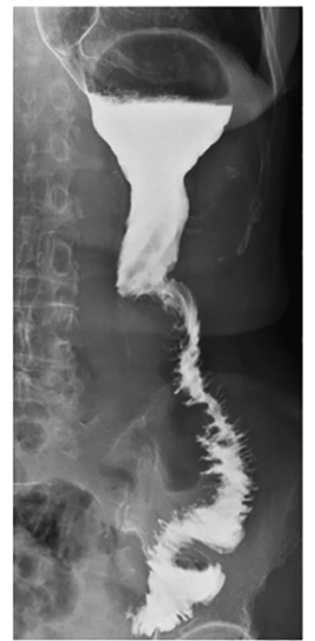

B

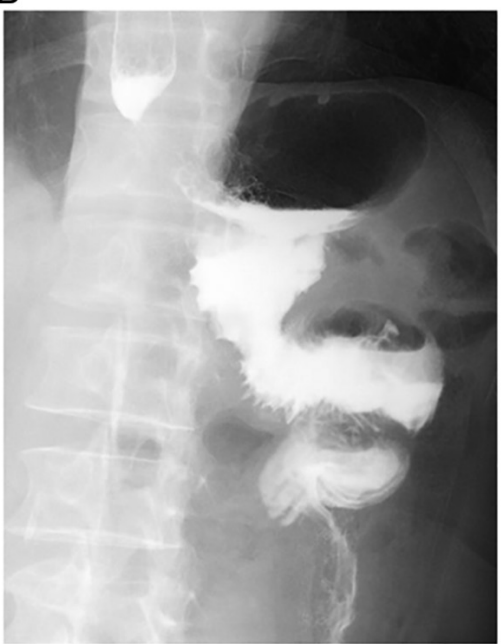

Figure 8. Upper gastrointestinal fluoroscopic images on postoperative day 10. There was no anastomotic failure or stenosis of the gastrojejunostomy in either (A) case 1 or (B) case 2.

there was no occurrence of other complications including internal hernia or ileus.

\section{Discussion}

Following TLDG, Billroth-II (B-II) or R-Y reconstruction is generally performed when the remnant stomach is small or the length of the duodenal bulb is short. We carry out R-Y reconstruction in this situation because it is thought to cause less bile reflux into the remnant stomach and esophagus 
A

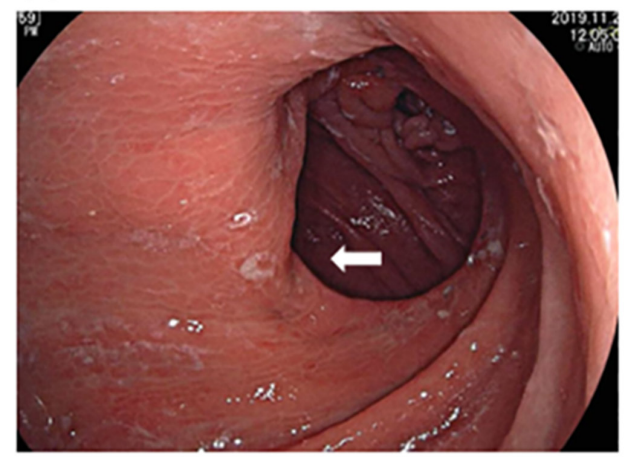

B

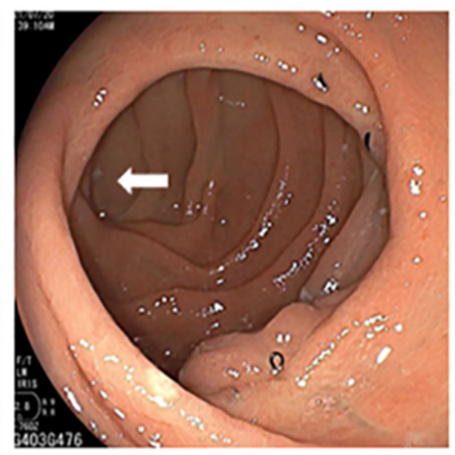

Figure 9. Endoscopic views of the gastrojejunostomies at 3 months after the operation. In both (A) case 1 and (B) case 2, the efferent loop (white arrow) was located on the patient's left side, and there was no twisting of the gastrojejunostomy and lifted jejunum postoperatively.

compared with B-II reconstruction $(13,14)$. In the present patients, no findings of reflux esophagitis and gastritis were encountered on endoscopic examination 3 months after the operation.

Several authors reported antecolic R-Y reconstruction with its efferent loop located on the patient's left side using only endoscopic linear staplers following TLDG $(1,4,5)$. In this gastrojejunostomy technique, first, without dividing the lifted jejunum, stapling of the greater curvature of the remnant stomach and the lifted jejunum without creating a gap is performed using one linear stapler so that the distal side of the lifted jejunum is located on the patient's left side, and closure of the stapler entry hole and division of the lifted jejunum are carried out simultaneously by a second stapling. In this procedure, twisting of the lifted jejunum when performing the gastrojejunostomy is prevented because the first stapling of the remnant stomach and lifted jejunum is performed without dividing the jejunum. Moreover, because the jejunal arteries diverge from the superior mesenteric artery toward the patient's left side, it is physiological that the distal side of the jejunum be located on the left side (9). Thus, twisting of the lifted jejunum after performing gastrojejunostomy is prevented when the efferent loop is located on the left side, and it is assumed that this reconstruction system prevents twisting of the linear-stapled gastrojejunostomy and lifted jejunum both intraoperatively and postoperatively (9).

However, because closure of the stapler entry hole and division of the lifted jejunum are performed simultaneously by a second stapling in this gastrojejunostomy technique, this second stapling for the posterior side of the entry hole tends to be uncertain due to the presence of the undivided lifted jejunum (4). Because anastomotic failure of the gastrojejunostomy can occur for this reason, we carried out the gastrojejunostomy in the present two patients by 1) creating the sacrificed jejunum from a portion $20 \mathrm{~cm}$ distal to the ligament of Treitz to a portion $30 \mathrm{~cm}$ distal; 2) dividing the sacrificed jejunum using two linear staplers; 3) retracting the knotted 3-0 Prolene suture on the anterior side of the stapler entry hole toward the ventral side to create a good view of the posterior side; 4) performing single-layer full-thickness and serosubmucosal continuous suturing with a $15-\mathrm{cm}-\mathrm{long}$ absorbable barbed suture (3-0 V-Loc 180) from the posterior side to the anterior side; and 5) adding several full-thickness knotted sutures of 3-0 Prolene on the site with a broad pitch. Irwin et al reported that a single-layer serosubmucosal anastomosis resulted in no incidence of anastomotic failure of the gastrojejunostomy (15), and no such failure occurred in the present patients.

Noshiro et al reported that when linear stapling of the greater curvature of the remnant stomach and the lifted jejunum is performed without dividing the jejunum in antecolic R-Y reconstruction with its efferent loop located on the patient's left side following TLDG, the outlet of the lifted jejunum is sometimes raised cranially, leading to bending of the lifted jejunum (3). As they indicated, when the remnant stomach is very small in antecolic R-Y reconstruction with its efferent loop located on the patient's left side following TLDG, linear stapling of the posterior wall of the remnant stomach and the lifted jejunum after dividing the jejunum and hand suturing of the stapler entry hole is considered to be advisable because the outlet of the lifted jejunum is arranged caudally, which prevents bending of the lifted jejunum (3).

In conclusion, intracorporeal gastrojejunostomy consisting of linear stapling and hand suturing can be one suggested option for gastrojejunostomy in antecolic R-Y reconstruction with its efferent loop located on the patient's left side following TLDG because this procedure can aid in the prevention of anastomotic failure.

\section{Acknowledgements}

Not applicable.

\section{Funding}

No funding was received.

\section{Availability of data and materials}

All data generated or analyzed during this study are included in this published article.

\section{Authors' contributions}

TaT and KY performed the operations. AO, ToT, and IK advised on patient treatment. TaT, KK, SH and KY obtained 
the medical images (e.g., upper gastrointestinal fluoroscopy and endoscopy). TaT and EN analyzed and interpreted the patient data regarding the surgical technique. TaT and EN confirm the authenticity of all the raw data. All authors have read and approved the final manuscript.

\section{Ethics approval and consent to participate}

This surgical procedure was approved by the Institutional Review Board of the Otori Stomach and Intestines Hospital (approval no. 19000010; Osaka, Japan) and Tanigawa Memorial Hospital (approval no. 21000001; Osaka, Japan). Written informed consent was obtained from the patients. The identities of the patients are fully anonymized in this report.

\section{Patient consent for publication}

Written informed consent was obtained from the patients for the publication of their data and images.

\section{Competing interests}

The authors declare that they have no competing interests.

\section{References}

1. Takaori K, Nomura E, Mabuchi H, Lee SW, Agui T, Miyamoto Y, Iwamoto M, Watanabe $\mathrm{H}$ and Tanigawa $\mathrm{N}$ : A secure technique of intracorporeal Roux-Y reconstruction after laparoscopic distal gastrectomy. Am J Surg 189: 178183, 2005.

2. Uyama I, Sakurai Y, Komori Y, Nakamura Y, Syoji M, Tonomura S, Yoshida I, Masui T, Inaba K and Ochiai M Laparoscopy-assisted uncut Roux-en-Y operation after distal gastrectomy for gastric cancer. Gastric Cancer 8: 253-257, 2005.

3. Noshiro H, Ohuchida K, Kawamoto M, Ishikawa M, Uchiyama A, Shimizu S and Tanaka M: Intraabdominal Roux-en-Y reconstruction with a novel stapling technique after laparoscopic distal gastrectomys totally laparoscopic gastrectomy. Gastric Cancer 12: 164-169, 2009.

4. Bouras G, Lee SW, Nomura E, Tokuhara T, Nitta T, Yoshinaka R, Tsunemi S and Tanigawa N: Surgical outcomes from laparoscopic distal gastrectomy and Roux-en-Y reconstruction: Evolution in a totally intracorporeal technique. Surg Laparosc Endosc Percutan Tech 21: 37-41, 2011.
5. Lee SW, Tanigawa N, Nomura E, Tokuhara T, Kawai M, Yokoyama K, Hiramatsu M, Okuda J and Uchiyama K: Benefits of intracorporeal gastrointestinal anastomosis following laparoscopic distal gastrectomy. World J Surg Oncol 10: 267, 2012.

6. Motoyama K, Kojima K, Hayashi M, Kato K, Inokuchi M and Sugihara K: $\beta$-Shaped intracorporeal Roux-en-Y reconstruction after totally laparoscopic distal gastrectomy. Gastric Cancer 17: 588-593, 2014

7. Ma JJ, Zang L, Yang A, Hu WG, Feng B, Dong F, Wang ML, Lu AG, Li JW and Zheng MH: A modified uncut Roux-en-Y anastomosis in totally laparoscopic distal gastrectomy: Preliminary results and initial experience. Surg Endosc 31: 4749-4755, 2017.

8. Nakamura K, Suda K, Suzuki A, Nakauchi M, Shibasaki S, Kikuchi K, Nakamura T, Kadoya S, Inaba K and Uyama I: Intracorporeal isosceles right triangle-shaped anastomosis in totally laparoscopic distal gastrectomy. Surg Laparosc Endosc Percutan Tech 28: 193-201, 2018.

9. Tokuhara T, Nakata E, Tenjo T, Kawai I, Kondo K, Ueda H and Tomioka A: Stenosis after esophagojejunostomy with the hemi-double-stapling technique using the transorally inserted anvil $\left(\mathrm{OrVil}{ }^{\mathrm{TM}}\right)$ in Roux-en-Y reconstruction with its efferent loop located on the patient's left side following laparoscopic total gastrectomy. Surg Endosc 33: 2128-2134, 2019.

10. Japanese Gastric Cancer Association: Japanese gastric cancer treatment guidelines 2018 (5th edition). Gastric Cancer 24: 1-21, 2021.

11. Tokuhara T, Nakata E, Tenjo T, Kawai I, Satoi S, Inoue K, Araki M, Ueda $\mathrm{H}$ and Higashi C: A novel option for preoperative endoscopic marking with India ink in totally laparoscopic distal gastrectomy for gastric cancer: A useful technique considering the morphological characteristics of the stomach. Mol Clin Oncol 6: 483-486, 2017

12. Hara K, Hayashi T, Nakazono M, Nagasawa S, Shimoda Y, Kumazu Y, Yamada T, Yamamoto N, Shiozawa M, Morinaga S, et al: An easy and reliable method to close Petersen's defect using barbed suture to prevent internal hernia from developing after gastrectomy with Roux-en-Y reconstruction. Asian J Endosc Surg 13: 238-241, 2020.

13. Fukuhara K, Osugi H, Takada N, Takemura M, Higashino M and Kinoshita H: Reconstructive procedure after distal gastrectomy for gastric cancer that best prevents duodenogastroesophageal reflux. World J Surg 26: 1452-1457, 2002.

14. Shim JH, Oh SI, Yoo HM, Jeon HM, Park CH and Song KY: Roux-en-Y gastrojejunostomy after totally laparoscopic distal gastrectomy: Comparison with Billorth II reconstruction. Surg Laparosc Endosc Percutan Tech 24: 448-451, 2014.

15. Irwin ST, Krukowski ZH and Matheson NA: Single layer anastomosis in the upper gastrointestinal tract. Br J Surg 77: 643-644, 1990. 